\title{
ORIGINAL
}

\section{DABIGATRAN VERSUS ACENOCUMAROL PARA LA PREVENCIÓN DEL ICTUS EN LA FIBRILACIÓN ATRIAL. ANÁLISIS DE IMPACTO PRESUPUESTARIO EN UN DEPARTAMENTO SANITARIO}

\author{
Álvaro Bonet Pla (1), Victoria Gosalbes Sóler (1), Manuel Ridao-López (2,3), Jorge Navarro Pérez (1), \\ Blanca Navarro Cubells (4) y Salvador Peiró (3).
}

(1) Centro de Salud Salvador Pau. Fundación de Investigación del Hospital Clínico Universitario - Instituto de Investigación Sanitaria (INCLIVA), Valencia.

(2) Instituto Aragonés de Ciencia de la Salud (IACS) - Instituto de Investigación Sanitaria Aragón (IISA), Zaragoza. Red de Investigación en Servicios de Salud en Enfermedades Crónicas (REDISSEC).

(3) Centro Superior de Investigación en Salud Pública (CSISP-FISABIO), Valencia. Red de Investigación en Servicios de Salud en Enfermedades Crónicas (REDISSEC).

(4) Servicio de Oncohematología, Hospital Clínico Universitario de Valencia. Fundación de Investigación del Hospital Clínico Universitario - Instituto de Investigación Sanitaria (INCLIVA), Valencia.

Financiación Ninguna. Conflicto de intereses y descargos Ninguno en relación con este manuscrito.

\begin{abstract}
RESUMEN
Fundamento: La aparición de nuevas opciones terapéuticas con diferentes efectividad y costes requiere la revaluación del papel de los actuales programas de anticoagulación oral (AO) para informar la toma de decisiones. El objetivo del trabajo es estimar el impacto presupuestario de la utilización generalizada de Dabigatrán a dosis de $110 \mathrm{mg}$ y $150 \mathrm{mg}$ en pacientes con fibrilación atrial (FA) respecto al escenario actual de tratamiento con acenocumarol.

Métodos: Cálculo del impacto presupuestario en 3 escenarios diferentes de anticoagulación oral: a) tratamiento con acenocumarol, b) sustitución generalizada de acenocumarol por Dabigatrán a dosis de $110 \mathrm{mg}$ y c) sustitución generalizada de acenocumarol por Dabigatrán a dosis de de $150 \mathrm{mg}$. El análisis se realizó desde la perspectiva de la Agencia Valenciana de Salud y con un horizonte temporal de 1 año (2009). La efectividad y los efectos adversos se extrapolaron del estudio RE-LY, mientras que los datos de prevalencia y costes procedieron de las estimaciones en el Departamento Sanitario.

Resultados: Se incluyó a 5.889 pacientes $(2,4 \%$ de la población $>18$ años) diagnosticados de FA de origen no valvular, de los que $3.726(63,2 \%)$ recibían tratamiento con acenocumarol. Los costes totales de los respectivos escenarios fueron de 1.119.412€ (300€ paciente/año) para acenocumarol, $4.985 .095 €$ (1.337€ paciente/año) para dabigatrán $110 \mathrm{mg}$ y $4.981 .226 €$ (1.336€ paciente/año) para dabigatrán $150 \mathrm{mg}$, con un impacto económico de 1.037 euros por paciente que cambiara de acenocumarol a dabigatrán 150 .

Conclusiones: El elevado impacto presupuestario de pasar a un escenario de sustitución generalizada de dicumarínicos a Dabigatrán apoya la restricción de esta estrategia terapéutica a subgrupos de pacientes de alto riesgo o de difícil control.
\end{abstract}

Palabras clave: Anticoagulantes orales. Dabigatrán. Acenocumarol. Costes de la atención médica. Fibrilación atrial.

\section{Correspondencia}

Salvador Peiró

Centro Superior de Investigación en Salud Pública

Av. Cataluña 21

46020 Valencia

peiro_bor@gva.es

\section{ABSTRACT}

\section{Dabigatran versus Acenocumarol for the Prevention of Stroke in Atrial Fibrillation. Budget Impact Analysis in one Health Department in Spain}

Background: To Estimate, in the context of a Health Department of the Valencia Health Agency, the budgetary impact of the widespread use of dabigatran at doses of 110 and $150 \mathrm{mg}$ in patients with non-valvular atrial fibrillation (AF), regarding the current scenario with acenocoumarol therapy.

Methods: Budget impact analysis of three scenarios of oral anticoagulation use in AF: a) current treatment with acenocoumarol, b) widespread replacement of acenocoumarol for Dabigatran $110 \mathrm{mg}$ and, c) idem at doses of $150 \mathrm{mg}$. The analysis was conducted from the perspective of the Valencia Health Agency with a time horizon of one year (2009). The effectiveness and adverse effects were extrapolated from the RE-LY study, while prevalence and cost data correspond to the Health Department estimates in 2009 .

Results: We included 5889 patients $(2.4 \%$ of the population $>18$ years) diagnosed with $\mathrm{AF}$, of which $3726(63.2 \%)$ were treated with acenocoumarol. The total costs of each scenario were $€ 1,119,412$ ( $€ 300$ patient/year) for acenocoumarol, $€ 4,985,095$ ( $€ 1,337$ patient/year) for dabigatran 110 and $€ 4,981,226$ ( $€ 1,336$ patient/year) for dabigatran 150 , with a budget impact of 1,037 euros/year per patient shifted from acenocumarol to dabigatran-150 .

Conclusions: The high budgetary impact of moving to a scenario of widespread substitution of warfarin for Dabigatran supports the restriction of this therapeutic strategy to subgroups of patients at high risk or difficult control.

Keywords: Anticoagulant. Dabigatran. Acenocoumarol. Health Care Costs. Atrial fibrillation. Economics. 


\section{INTRODUCCIÓN}

La fibrilación auricular (FA) es un trastorno del ritmo cardiaco de gran relevancia tanto por su elevada prevalencia $(8,5 \%$ en personas mayores de 60 años y más del $15 \%$ en personas mayores de 80 años $)^{1}$ como por el riesgo trombótico que comporta (un 20\% de los ictus se deben a embolismos por FA) y su gravedad, que es superior que la de otros ictus ${ }^{2,3}$. El manejo convencional de los pacientes con FA incluye el control del ritmo cardíaco, de los factores de riesgo cardiovascular y la prevención de los episodios tromboembólicos con anticoagulantes orales o antiagregantes plaquetarios $^{4}$ si la valoración en las escalas de riesgo lo aconseja. Realizada tradicionalmente con fármacos del grupo de los dicumarínicos, la anticoagulación oral (AO) reduce la morbimortalidad y es superior a otros tratamientos en la prevención del ictus asociado a $\mathrm{FA}^{5}$. La AO se aconseja en pacientes de alto riesgo tromboembólico ${ }^{6}$ cuando los beneficios esperables superan los riesgos de las complicaciones hemorrágicas, aunque en los últimos años se han modificado las escalas de este riesgo para recomendar AO a pacientes que antes no la recibían $\left(\mathrm{CHAD}_{2} \geq 2\right)^{7}$.

Los dicumarínicos tienen una ventana terapéutica estrecha por debajo de la cual se reduce la protección de la embolia y por encima se incrementa el riesgo de hemorragia, hay que ajustarlos en cada paciente y, además, tienen múltiples interacciones con otros medicamentos y con algunos alimentos. Por ello, su manejo requiere monitorización continua - cada 3-5 semanasmediante el control analítico del International Normalized Ratio (INR). En la mayoría de países este seguimiento se realiza en programas de control de anticoagulación por profesionales de atención primaria y de los servicios de hematología hospitalarios, que en las evaluaciones económicas realizadas han sido valorados como costo-efectivos $^{8,9}$. La comercialización de nuevos anticoagulantes orales ${ }^{10}$ que inhiben de forma directa el factor II (dabigatrán) o el factor X (rivaroxabán, apixaban) ha modificado sustancialmente el arsenal terapéutico de la $\mathrm{AO}$ y los nuevos fármacos se postulan como alternativa a los dicumarínicos con la gran ventaja añadida de que su perfil farmacocinético estable hace innecesaria la monitorización regular del efecto anticoagulante. Pero con la desventaja, también importante, del notable mayor precio del fármaco.

La eficacia comparativa de dabigatrán frente a warfarina en pacientes con FA fue evaluada en el estudio RE-LY ${ }^{11}$, un extenso ensayo clínico aleatorizado, sin enmascaramiento, de no inferioridad, en el que se valoró la eficacia de las dosis de $110 \mathrm{mg}$ y $150 \mathrm{mg}$ para reducir la incidencia de ictus isquémico, así como su seguridad, definida por la incidencia de complicaciones hemorrágicas. Los resultados de la eficacia fueron similares a los de warfarina para la dosis de $110 \mathrm{mg}$ (aunque con menor incidencia de complicaciones hemorrágicas, sobre todo cerebrales), mientras que a dosis de $150 \mathrm{mg}$ se obtuvo una reducción significativa de los ictus pero con tasas de sangrado grave comparables a las de warfarina.

La aparición de estas nuevas opciones terapéuticas, diferentes en eficacia y costes, requiere la revaluación del papel de los actuales programas de $\mathrm{AO}$ para informar la toma de decisiones sobre si mantener la anticoagulación tradicional con dicumarínicos (que en España están representados fundamentalmente por el acenocumarol) o cambiar a los nuevos fármacos. En esta toma de decisiones, además de la eficacia y seguridad de cada fármaco en las condiciones controladas de un ensayo clínico, conviene tener en cuenta su efectividad en la práctica habitual de cada organización sanitaria, la eficiencia de ambas alternativas (el coste adicional por unidad de beneficio adicional, es decir si desde el punto de 
vista de la sociedad, el fármaco "vale" más de lo que cuesta) y su impacto presupuestario: el coste neto para el sistema sanitario (que no solo incluye el precio del medicamento, sino cualquier otro que repercuta sobre los presupuestos sanitarios, incluyendo los costes de las complicaciones o los costes evitados por la reducción de eventos adversos). El análisis de impacto presupuestario plantea la pregunta de si podemos pagar o no el nuevo fármaco (una pregunta que, formalmente, sólo se debería plantear cuando el fármaco ha mostrado ser coste-efectivo). Adicionalmente, el análisis de impacto presupuestario muestra los costes de oportunidad para el financiador público, ya que cuantifica los recursos que no podrán destinarse a otros fines sanitarios y, en consecuencia, plantea la pregunta de si la organización sanitaria podría obtener más beneficios clínicos con estos recursos destinados a otra finalidad ${ }^{12}$.

Los análisis de eficiencia e impacto presupuestario, más allá de los resultados de los estudios económicos realizados en otros entornos (con distintos sistemas sanitarios, organización diferente y costes muy diferentes, tanto para los tratamientos como para la morbilidad evitada), deben considerar la prevalencia, grado de control, organización y costes de cada contexto concreto.

El objetivo del trabajo es estimar el impacto presupuestario de la utilización de Dabigatrán a dosis de 110 y $150 \mathrm{mg}$ en pacientes con fibrilación atrial (FA) respecto al escenario actual de tratamiento con acenocumarol.

\section{MATERIAL Y MÉTODOS}

Diseño. Se establecieron 3 escenarios de uso de la $\mathrm{AO}$ en la prevención del ictus isquémico en pacientes con FA: a) tratamiento con acenocumarol, b) sustitución por Dabigatrán a dosis de $110 \mathrm{mg} \mathrm{y} \mathrm{c)} \mathrm{sus-}$ titución por Dabigatrán a dosis de $150 \mathrm{mg}$ (figura 1). El análisis se realizó desde la perspectiva de la Agencia Valenciana de Salud con un horizonte temporal de 1 año. Todos los datos están referidos a 2009.

Ámbito. Departamento Sanitario Hospital Clínico-Malvarrosa de Valencia, que cuenta con 16 zonas básicas de salud (ZBS) y 32 puntos de atención entre centros de salud y consultorios auxiliares. Debido a la coexistencia de varios modelos de organización de la AO, el estudio utilizó datos exclusivamente de 12 de las 16 zonas de salud, que atendían a un total de 237.730 personas mayores de 18 años y desarrollaban el modelo de control AO más extendido en el Departamento, consistente en la punción digital en el centro de salud y dosificación centralizada por hematólogos en la unidad de anticoagulación del Hospital del Departamento.

Medidas de la efectividad. La medida principal de efectividad fue la incidencia diferencial de infartos cerebrales en cada alternativa, extrapolados de los resultados del estudio RE-LY ${ }^{11}$ y ajustados a un año de seguimiento. También se consideró la incidencia de los principales efectos adversos del tratamiento anticoagulante: hemorragias intracraneales, digestivas y el infarto agudo de miocardio.

Identificación de costes. En la alternativa con acenocumarol se consideraron los costes del fármaco, los derivados de la extracción de la muestra para la determinación del INR en los centros de salud y la posterior transmisión de datos mediante sistema informático al servicio de hematología del hospital (donde se centraliza la prescripción de dosis, con recuperación de la información de la pauta de tratamiento hasta el próximo control por parte del personal de enfermería del centro de salud).

En las alternativas con dabigatrán se calcularon los costes que supondría el tratamiento de los pacientes a las dosis de 110 
Figura 1

Árbol de decisión

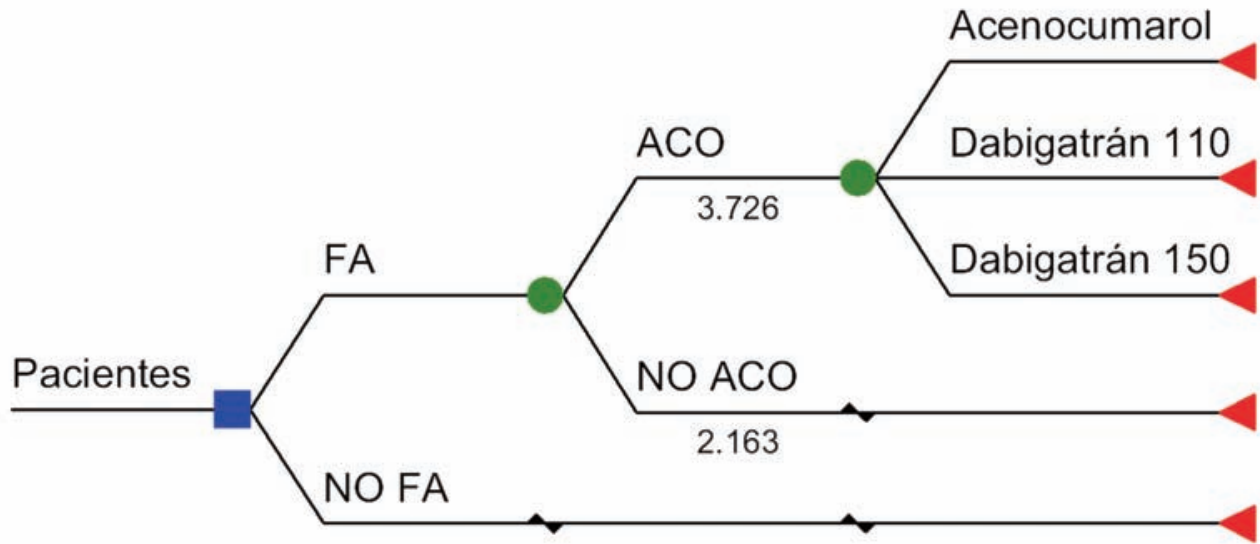

mg y $150 \mathrm{mg}$. En ambas se incluyeron los costes hospitalarios de la atención a los infartos cerebrales no prevenidos así como los costes de hospitalización de las hemorragias intracraneales, digestivas y del infarto agudo de miocardio.

Cuantificación de los costes. Se determinó el coste total y el coste por paciente/año de cada uno de los parámetros analizados. En primer lugar se calcularon los costes del tratamiento farmacológico, determinándose los $\mathrm{mg}$ de acenocumarol prescritos a los pacientes (dosis media de $16 \mathrm{mgr} / \mathrm{semana}$ ) y multiplicándolos por el

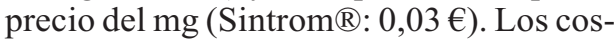
tes del tratamiento con Dabigatrán se calcularon a partir del Precio de Venta al Público (PVP) autorizado en España (Pradaxa ${ }^{\circledR}: 98,35 €$ para la caja de 60 comprimidos, tanto de la presentación de $110 \mathrm{mg}$ como de la de $150 \mathrm{mg}$ ) y considerando la posología recomendada (1 comprimido cada 12 horas). A continuación se obtuvieron los costes de los eventos clínicos, infartos cerebrales como resultado primario y de las complicaciones fundamentales a partir de los costes de los Grupos de Diagnósticos Relacionados (GDR) 810, 170 y 15 en el Hospital del Departamento. Adicionalmente, sólo para acenocumarol, se obtuvieron los costes de los reactivos empleados para los controles analíticos (Protime 3 y Tenderlett Plus LV) multiplicando el número de determinaciones realizadas por el precio ( $3,6 €$ por kit) de cada determinación, así como los costes del personal implicado en el programa de control del tratamiento anticoagulante (auxiliar administrativo, enfermería y hematólogía). Los tiempos se estimaron como la media diaria dedicada por cada profesional a esa tarea a partir de los tiempos de dedicación declarados por los responsables de cada servicio y centros de salud (tabla 1). Los datos de costes hospitalarios por GDR y de personal fueron facilitados por la Dirección del Departamento Clínico-Malvarrosa para el año 2009.

Análisis estadístico. El impacto presupuestario de la utilización generalizada de dabigatrán $110 \mathrm{mg}$ y $150 \mathrm{mg}$ se estimó como el coste incremental neto para el sistema sanitario (gasto adicional menos ahorros por reducción de eventos) respecto al escenario base configurado por el coste del tratamiento con acenocumarol. Adicionalmente, se estimó el coste incremental por ictus evitado en cada uno de los escenarios con Dabigatrán respecto al escenario base. 
Tabla 1

Estimación tiempos de dedicación de personal al control de la International Normalized Ratio (INR)

\begin{tabular}{|c|c|c|c|c|c|}
\hline & \multicolumn{2}{|c|}{ Administrativo } & \multicolumn{2}{|c|}{ Enfermería } & \multirow{2}{*}{\begin{tabular}{|c|} 
Hematólogos \\
Hospital
\end{tabular}} \\
\hline & Hospital & Primaria & Hospital & Primaria & \\
\hline Minutos/día & 180 & 70 & 60 & 2.617 & 540 \\
\hline Horas/año & 792 & 308 & 264 & 11.515 & 2376 \\
\hline Meses/año & 5,1 & 2 & 1,7 & 75 & 15,4 \\
\hline TOTAL meses/año & \multicolumn{2}{|c|}{7,1} & \multicolumn{2}{|c|}{76,5} & 15,4 \\
\hline Salario Medio & \multicolumn{2}{|c|}{1.284} & \multicolumn{2}{|c|}{2.130} & 3.206 \\
\hline
\end{tabular}

\section{RESULTADOS}

De los 5.889 pacientes diagnosticados de FA no valvular $(2,47 \%$ de la población mayor de 18 años) en las zonas de salud incluidas en el estudio, $3.726(63,2 \%)$ estaban en tratamiento con AO (tabla 2). El número de ictus esperable en esta población (tabla 3) se estimó en 59 para la alternativa con acenocumarol, 54 (-5 respecto a acenocumarol) en la alternativa tratamiento con Dabigatrán 110 mg y 38 en la alternativa Dabigatrán 150mg (-21 respecto a acenocumarol y -16 respecto a Dabigatran $110 \mathrm{mg}$ ). Las hemorragias intracraneales se estimaron en 28,8 y 12 respectivamente, las hemorragias digestivas en 40, 43 y 58 respectivamente y los casos de infarto agudo de miocardio (IAM) en 24, 24 y 30 respectivamente para Acenocumarol, Dabigatran-110 y Dabigatran-150 (tabla 3).

Los costes anuales del tratamiento farmacológico se estimaron en $447.092 €$ para el Acenocumarol (incluyendo personal, reactivos y fármaco) y en 4.456.296€ tanto para el tratamiento con dabigatrán $110 \mathrm{mg}$ como con Dabigatrán 150 mg (tabla 4). Respecto a los ictus esperados en cada alternativa, los costes hospitalarios fueron de $310.531 €$ (acenocumarol), 283.016€ (dabigatrán 110 mg) y $198.504 €$ (dabigatrán $150 \mathrm{mg}$ ). Al añadir los costes de los efectos adversos, los costes hospitalarios ascendieron a 672.663 $€$ (acenocumarol), $528.797 €$ (dabigatrán
$110 \mathrm{mg}$ ) y $524.929 €$ (dabigatrán $150 \mathrm{mg}$ ). Los costes totales para el Departamento serían 1.119.412 $€$ en la alternativa Acenocumarol, 4.985.095 $€$ y 4.981.266 $€$ en las alternativas con dabigatrán 110 y $150 \mathrm{mg}$ respectivamente.

El impacto económico de pasar del tratamiento con acenocumarol a Dabigatrán a todos los pacientes (tabla 5) sería de $3.865 .683 €$ para dabigatrán 110 y de $3.861 .854 €$ para dabigatrán 150 . Los costes

Tabla 2

\section{Población y pacientes con fibrilación atrial y anticoagulación oral}

\begin{tabular}{|c|c|c|}
\hline ZONA & $\begin{array}{c}\text { Población } \\
>18 \text { años }\end{array}$ & $\begin{array}{c}\text { Pacientes con AO } \\
(\mathrm{n}(\%)\end{array}$ \\
\hline ZONA 1 & 17.132 & $268(1,56)$ \\
\hline ZONA 3 & 11.030 & $198(1,80)$ \\
\hline ZONA 4 & 18.887 & $367(1,94)$ \\
\hline ZONA 5 & 9.018 & $157(1,74)$ \\
\hline ZONA 6 & 10.488 & $179(1,71)$ \\
\hline ZONA 8 & 8.231 & $123(1,49)$ \\
\hline ZONA 9 & 34.126 & $441(1,29)$ \\
\hline ZONA 10 & 32.833 & $564(1,72)$ \\
\hline ZONA 11 & 34.375 & $447(1,30)$ \\
\hline ZONA 13 & 21.856 & $272(1,24)$ \\
\hline ZONA 15 & 32.344 & $585(1,81)$ \\
\hline ZONA 16 & 7.410 & $125(1,69)$ \\
\hline TOTAL & 237.730 & $3.726(1,57)$ \\
\hline
\end{tabular}




\section{Tabla 3}

Incidencia y número de casos esperados en un año de seguimiento de ictus, hemorragia craneal, hemorragia digestiva e infarto de miocardio en las alternativas de tratamiento

\begin{tabular}{|c|c|c|c|c|c|c|c|c|}
\hline & \multicolumn{2}{|c|}{$\begin{array}{c}\text { Ictus } \\
\text { trombótico }\end{array}$} & \multicolumn{2}{c|}{$\begin{array}{c}\text { Hemorragia } \\
\text { craneal }\end{array}$} & \multicolumn{2}{c|}{$\begin{array}{c}\text { Hemorragia } \\
\text { Digestiva }\end{array}$} & \multicolumn{2}{c|}{$\begin{array}{c}\text { Infarto agudo } \\
\text { miocardio }\end{array}$} \\
\cline { 2 - 10 } & Incidencia & $\mathrm{n}$ & Incidencia & $\mathrm{n}$ & Incidencia & $\mathrm{n}$ & Incidencia & $\mathrm{n}$ \\
\hline Acenocumarol & $1,58 \%$ & 59 & $0,76 \%$ & 28 & $1,08 \%$ & 40 & $0,64 \%$ & 24 \\
\hline Dabigatran $110 \mathrm{mg}$ & $1,44 \%$ & 54 & $0,23 \%$ & 8 & $1,15 \%$ & 43 & $0,64 \%$ & 24 \\
\hline Dabigatran $150 \mathrm{mg}$ & $1,01 \%$ & 38 & $0,32 \%$ & 12 & $1,56 \%$ & 58 & $0,81 \%$ & 30 \\
\hline
\end{tabular}

unitarios anuales por paciente anti-coagulado serían de $300 €$ (acenocumarol), $1.338 €$ (dabigatrán 110) y $1.337 €$ (dabigatrán 150), con un impacto presupuestario discretamente superior a los 1.000 euros anuales por paciente que cambiara de acenocumarol a dabigatrán. El análisis incremental muestra un coste de $181.833 €$ por ictus evitado al pasar a la alternativa dabigatran $150 \mathrm{mg}$ y de $741.063 €$ en la alternativa dabigatrán 110 , que queda dominada respecto a Dabigatrán 150 al presentar costes similares pero menor efectividad.

Tabla 4

Costes totales y por paciente del uso de acenocumarol, dabigatran-110 mg y dabigatrán $150 \mathrm{mg}$ en un Departamento de Salud

\begin{tabular}{|c|c|c|c|}
\hline & Acenocumarol & Dabigatrán-110 & Dabigatran-150 \\
\hline \multicolumn{4}{|c|}{ Coste anual del tratamiento farmacológico } \\
\hline Coste administrativos & 8.942 & 0 & 0 \\
\hline Coste enfermería & 162.862 & 0 & 0 \\
\hline Coste hematólogos & 49.184 & 0 & 0 \\
\hline Coste reactivos* & 132.954 & 0 & 0 \\
\hline Coste fármacos & 93.150 & 4.456 .296 & 4.456 .296 \\
\hline Total costes tratamiento AO & 447.092 & 4.456 .296 & 4.456 .296 \\
\hline \multicolumn{4}{|c|}{ Coste anual del tratamiento de los ictus y los efectos adversos } \\
\hline Coste ictus & 310.531 & 283.016 & 198.504 \\
\hline Coste hemorragia intracraneal & 175.189 & 53.017 & 73.764 \\
\hline Coste hemorragia digestiva & 89.817 & 95.638 & 129.736 \\
\hline Coste infarto miocardio & 97.126 & 97.126 & 122.925 \\
\hline Total ictus y complicaciones & 672.663 & 528.797 & 524.929 \\
\hline \multicolumn{4}{|c|}{ Costes unitarios anuales por paciente en AO } \\
\hline Coste fármacos & 25 & 1.196 & 1.196 \\
\hline Coste tratamiento exc. fármacos & 95 & 0 & 0 \\
\hline Coste ictus y efectos adversos & 180 & 142 & 141 \\
\hline Coste total & 300 & 1.338 & 1.337 \\
\hline
\end{tabular}

AO: antiocoagulación oral; *3,6 (precio reactivo) por 9,9 determinaciones por paciente/año. 
Tabla 5

Impacto presupuestario y coste incremental por ictus evitado

\begin{tabular}{|l|c|c|c|}
\hline & $\begin{array}{c}\text { Escenario base } \\
\text { (acenocumarol) }\end{array}$ & dabigatrán $110 \mathrm{mg}$ & Dabigatran $150 \mathrm{mg}$ \\
\hline Coste total en cada escenario & 1.119 .412 & 4.985 .095 & 4.981 .266 \\
\hline Coste TOTAL & - & 3.865 .683 & 3.861 .854 \\
\hline Impacto presupuestario & 300 & 1.338 & 1.337 \\
\hline Costes unitarios por paciente con AO & - & 1.038 & 1.037 \\
\hline Coste total & & & \\
\hline Impacto presupuestario & 0 & 5 & 21 \\
\hline Coste incremental por ictus evitado & - & 741.063 & 181.833 \\
\hline Ictus evitados & & \\
\hline Coste ictus evitado & &
\end{tabular}

\section{DISCUSIÓN}

Los resultados de este estudio, realizado con datos de costes de un Departamento de Salud y asumiendo la efectividad de los tratamientos del estudio RELY, muestran que generalizar el tratamiento con dabigatrán $150 \mathrm{mg}$ en el Departamento estudiado supondría un coste neto anual de 3,86 millones de euros para el sistema sanitario público de la Comunidad Valenciana, unos 1.000 euros adicionales por paciente tratado y en torno a 180.000 euros por ictus evitado. Aceptando que estos datos fueran generalizables a otros territorios, el impacto presupuestario se situaría en unos 65 millones de euros en la Comunidad Valenciana (el 1\% del presupuesto sanitario de la Generalitat en 2009) y unos 600 millones de euros para el conjunto del SNS (en torno al 5\% del gasto farmacéutico en receta oficial del año 2009). Ajustando por población, una cifra próxima a los $700 £$ millones estimados en Reino Unido para un escenario de sustitución generalizada de los dicumarínicos por dabigatrán ${ }^{13}$. Este alto impacto presupuestario es consecuencia, fundamentalmente, del elevado precio de dabigatrán (1.196€ por paciente y año) en relación al del acenocumarol (120€ por paciente y año, incluyendo la monitoriza- ción), que no alcanza a ser compensado por los ahorros potenciales derivados de la reducción de los eventos primarios (aplicable sólo a la dosis de 150) o de las complicaciones hemorrágicas cerebrales.

Estos resultados no son contradictorios sino que complementan los de los estudios coste-efectividad que tienden a valorar dabigatran $150 \mathrm{mg}$ (no tanto Dabigatrán 110 mg, que quedaría relegado a aquellos casos que requirieran una dosificación menor, como en la insuficiencia renal) como una opción costeefectiva (esto es, por debajo de los umbrales de coste por AVAC usados comúnmente en cada país para definir si una tecnología es coste-efectiva) en pacientes de riesgo alto $\left(\mathrm{CHAD}_{2} \geq 2\right.$ ó 3 según trabajos) salvo un control excelente del INR ${ }^{14-17}$. En estos estudios, el coste por AVAC crece notablemente cuando el paciente es de bajo riesgo o cuando el control del INR es adecuado (aunque no tanto en las evaluaciones económicas asociadas al fabricante de dabigatrán, que parecen usar una misma modelización de efectividad basada en el RE-LY -aun adaptando los costes a cada país-y en las que incluso en los pacientes de bajo riesgo el coste por AVAC tiende a mantenerse por debajo de los dinteles de coste-efectividad habituales) $)^{18-21}$. 
Esta situación de tecnología coste-efectiva (al menos para algunos subgrupos de pacientes) e impacto presupuestario muy elevado, ha llevado a las instituciones responsables de la incorporación de medicamentos a la cartera de los servicios públicos de diferentes países a limitar su uso a los pacientes de alto riesgo o que no consiguen un buen control del INR ${ }^{22-25}$. Estas recomendaciones coinciden notablemente con las realizadas recientemente por la Agencia Española de Medicamentos y Productos Sanitarios ${ }^{26}$ sin utilizar, al menos explícitamente, los análisis coste-efectividad o de impacto presupuestario y reducirían al menos en dos tercios la población de candidatos a tratamiento con los nuevos anticoagulantes (y el impacto presupuestario de su uso, además de mejorar la relación costeefectividad al seleccionar los pacientes con mayores beneficios esperables).

En todo caso, las decisiones sobre la utilización de dabigatrán (y los nuevos anticoagulantes en general) deberían considerar una serie de factores que no son tenidos en cuenta en los análisis económicos habituales:

1. La incertidumbre sobre los efectos adversos de los nuevos AO, dada la escasa experiencia con estos fármacos en relación con los dicumarínicos, con especial interés en el posible incremento de infartos de miocardio respecto a los dicumarínicos ${ }^{27}$.

2. La incertidumbre sobre la necesidad de controles analíticos o de otro tipo para monitorizar la seguridad de dabigatrán, como los controles de función renal (ya recomendados por la AEMPS $^{26}$ y otras Agencias de Medicamentos), hepáticos u otros, que encarecerían los costes de los escenarios con Dabigatrán.

3. La incertidumbre sobre el efecto de una posible baja adherencia de los nuevos $\mathrm{AO}$ en los resultados clínicos, acrecentada por la posibilidad de que el paso a 2 dosis por día (frente a 1 con acenocumarol) pudiera aumentar los olvidos de dosis. También se apunta que la ausencia de un dato objetivo y repetido como es el INR pueda hacer que el paciente se sienta menos "comprometido" con el tratamiento y lo abandone con más facilidad ${ }^{28}$ En el mismo sentido, el recientemente introducido copago para pensionistas y la duplicación del tope de precio en los medicamentos crónicos también podría contribuir a la pérdida de adherencia a dabigatrán, mientras que prácticamente no afectaría a acenocumarol, dado su bajo precio.

4. La posibilidad de que las mejoras en comodidad del manejo lleven a los clínicos a indicar esta terapia en pacientes en los que hasta ahora eran reticentes a iniciar el tratamiento con dicumarínicos.

5. La posibilidad de acuerdos de riesgo compartido entre los servicios de salud públicos y los fabricantes de nuevos anticoagulantes para facilitar el acceso -a precios más reducidos- de algunos subgrupos de pacientes a estos tratamientos. Probablemente existe un importante espacio para encontrar acuerdos precio-volumen que pudieran beneficiar al financiador, a los pacientes y a los propios fabricantes.

6. La posibilidad de desarrollar políticas de bajo coste de mejora de la calidad del manejo de los dicumarínicos, ya que una mejora sustantiva en el porcentaje de pacientes en rango INR reduciría considerablemente la ventaja en efectividad de los nuevos AO y existe una gran variabilidad entre centros en los resultados obtenidos en este aspecto.

7. En el caso de que la indicación de los nuevos AO se restrinja a los pacientes de mayor riesgo o no controlados, la puesta en marcha de las estrategias efectivas necesarias para prevenir que a los pacientes que no cumplen los criterios para cambiar de fármaco no se les pueda prescribir. 
Entre las limitaciones de este estudio hay que citar, en primer lugar, el horizonte temporal, limitado a un solo año. Los estudios de impacto presupuestario típicos consideran escenarios de 3 a 5 años para dar cabida a las variaciones en incidencia, un aspecto no tan relevante en nuestro estudio $^{12}$. En todo caso, el previsible incremento de la incidencia de FA y de sujetos tratados incrementaría el diferencial entre alternativas ya que buena parte de los costes de monitorización del INR son fijos, mientras que los costes del tratamiento farmacológico son variables y directamente asociados al número de pacientes. En segundo lugar, existe una notable variabilidad entre territorios, incluso a nivel del Sistema Nacional de Salud, en la proporción de pacientes diagnosticados de FA, la proporción de tratados, los costes de monitorización y los costes del manejo de los ictus y los sucesos adversos, por lo que los resultados de este estudio no son directamente extrapolables a otros entornos. No obstante, dadas las diferencias halladas, es muy improbable que las alteraciones en los costes (de la monitorización, de los ingresos hospitalarios) puedan modificar sustancialmente las conclusiones del estudio. En tercer lugar, los costes de los efectos adversos se han considerado similares entre alternativas, pero podrían ser diferentes, ya que dabigatrán no dispone de un antídoto específico y parece que sólo la diálisis consigue revertir su efecto anticoagulante $^{29}$. En cuarto lugar, se ha utilizado el PVP para calcular los costes de los fármacos cuando, en puridad, debería haberse considerado solamente el coste para la AVS, esto es, sin incluir los copagos a cargo del paciente. En el momento en que se realizó el estudio este aspecto era distinto (los pensionistas no tenían copago y para los activos existía un tope de 2,5 euros por envase). Con la reciente instauración del copago, en torno al 5\% de los costes de Dabigatrán sería asumido por el paciente, siendo irrelevante el impacto en el acenocumarol. Aunque se trata de una magnitud importante (casi 60 euros por paciente y año) no modificaría de forma sustantiva los resultados del análisis.

Siguiendo con las limitaciones, nuestro estudio utilizó los datos de efectividad media del estudio RE-LY ${ }^{11}$ pero el análisis por estratos de este estudio mostraba que la efectividad (reducción de ictus) en el grupo de los dicumarínicos era muy dependiente del grado de control, desapareciendo la ventaja de dabigatrán en los pacientes bien controlados con warfarina ${ }^{30}$. En este sentido, los ahorros derivados de la reducción de ictus dependen fundamentalmente del grado de control conseguido en cada Departamento y podrían ser menores si la calidad de la atención a los sujetos con AO fuera muy elevada. Por otra parte, en nuestro estudio (y en muchos otros trabajos ${ }^{21}$ ) se han extrapolado los resultados del control con warfarina al acenocumarol. Aunque ambos fármacos sean equivalentes en muchos aspectos, su farmacocinética es diferente (la vida media del acenocumarol es de 8-11 horas, frente a 31-51 para warfarina) y el porcentaje de pacientes en rango podría ser diferente según fármacos, afectando a la reducción de eventos y a las hemorragias ${ }^{31}$. Finalmente, nuestro estudio no es un análisis coste-efectividad. No valora el coste incremental por AVAC incremental, sino el impacto sobre los presupuestos sanitarios. En este sentido, no permite valorar la eficiencia del tratamiento con dabigatrán sino que describe el impacto de la estrategia de trasladar al conjunto de pacientes al tratamiento con este fármaco, permitiendo informar sobre la eficiencia de esta política frente a otras posibles.

Aunque los análisis de impacto presupuestario deberían utilizarse de forma habitual para mejorar la toma de decisiones sobre la inclusión o no de nuevos medicamentos, tecnologías y prestaciones en la cartera de servicios del SNS, en la actual situación de crisis económica y res- 
tricciones en los presupuestos sanitarios, su necesidad es aun más imperiosa. Se trata de valorar el impacto en los presupuestos sanitarios de diferentes escenarios de incorporación de estas tecnologías (siempre con medicamentos o tecnologías que han mostrado previamente su eficacia, seguridad y coste-efectividad) para ayudar a adoptar aquellas decisiones que generen mayores beneficios para los pacientes a partir de los recursos disponibles.

Nuestro trabajo muestra los elevados costes de pasar a un escenario de sustitución generalizada de dicumarínicos a dabigatrán, apoya la restricción de esta política terapéutica a los subgrupos de pacientes que más puedan beneficiarse (previsiblemente aquellos de difícil control del INR con porcentaje de tiempo en rango terapéutico menor del $65 \%$ o con antecedente de hemorragia intracraneal, en este caso a dosis de $110 \mathrm{mg}$ ) y, también, sugiere la posibilidad de buscar formas alternativas de mejorar el control de la AO que permitan incrementar la efectividad de estos programas a menor coste (programas de enfermería, de educación de los pacientes, de automanejo $\mathrm{u}$ otras). También sugiere que si los fabricantes de AO quieren incrementar el número de pacientes tratados deberían considerar la posibilidad de reducir su precio, bien directamente bien mediante acuerdos de riesgos compartidos en los que de alguna forma el incremento en el volumen de pacientes tratados conlleve una reducción del coste del tratamiento. En este sentido, la información aportada en este trabajo es relevante, tanto para los clínicos (que deberían evitar derrochar recursos en el manejo de los pacientes de bajo riesgo o con INR en rango, a fin de salvaguardarlos para otros pacientes), como para las administraciones sanitarias, que deberían diseñar las políticas adecuadas para enfrentar este problema antes que consentir un deslizamiento paulatino y sin criterios homogéneos de unos tratamientos a otros.

\section{BIBLIOGRAFÍA}

1. Cea-Calvo L, Redón J, Lozano JV, Fernández-Pérez C, Martí-Canales JC, Llisterri JL, et al. Prevalence of atrial fibrillation in the Spanish population aged 60 years or more. The PREV-ICTUS study. Rev Esp Cardiol. 2007;60(6):616-24.

2. Steger C, Pratter A, Martinek-Bregel M, Avanzini M, Valentin A, Slany J, et al. Stroke patients with atrial fibrillation have a worse prognosis than patients without: data from the Austrian Stroke registry. Eur Heart J. 2004;25(19):1734-40.

3. Lamassa M, Di Carlo A, Pracucci G, Basile AM, Trefoloni G, Vanni P, et al. Characteristics, outcome, and care of stroke associated with atrial fibrillation in Europe: data from a multicenter multinational hospital-based registry (The European Community Stroke Project). Stroke. 2001;32(2):392-8.

4. Fuster V, Rydén LE, Cannom DS, Crijns HJ, Curtis $\mathrm{AB}$, Ellenbogen KA, et al. $2011 \mathrm{ACCF} / \mathrm{AHA} / \mathrm{HRS}$ focused updates incorporated into the ACC/AHA/ESC 2006 Guidelines for the management of patients with atrial fibrillation: a report of the American College of Cardiology Foundation/American Heart Association Task Force on Practice Guidelines developed in partnership with the European Society of Cardiology and in collaboration with the European Heart Rhythm Association and the Heart Rhythm Society. J Am Coll Cardiol. 2011;57(11):e101-98.

5. Hart RG, Pearce LA, Aguilar MI. Meta-analysis: antithrombotic therapy to prevent stroke in patients who have Non-valvular atrial fibrillation. Ann Intern Med. 2007;146(12):857-67.

6. Gage BF, Waterman AD, Shannon W, Boechler M, Rich MW, Radford MJ. Validation of clinical classification schemes for predicting stroke: results from the National Registry of Atrial Fibrillation. JAMA. 2001;285(22):2864-70.

7. Lip GY, Nieuwlaat R, Pisters R, Lane DA, Crijns HJ. Refining clinical risk stratification for predicting stroke and thromboembolism in atrial fibrillation using a novel risk factor-based approach: the Euro Heart Survey on atrial fibrillation. Chest. 2010;137(2):263-72.

8. Navarro JL, César JM, Fernández MA, Fontcuberta J, Reverter J, Gol-Freixas J. Tratamiento anticoagulante oral. Estudio coste/beneficio. Rev Adm Sanit. 2008;6(3):525-42.

9. Connock M, Stevens C, Fry-Smith A, Jowett S, Fitzmaurice D, Moore D, et al. Clinical effectiveness and 
cost-effectiveness of different models of managing long-term oral anticoagulation therapy: a systematic review and economic modelling. Health Technol Assess. 2007;11(38):iii-iv, ix-66.

10. Eikelboom JW, Weitz JI. New anticoagulants. Circulation. 2010;121(13):1523-326.

11. Connolly SJ, Ezekowitz MD, Yusuf S, Eikelboom J, Oldgren J, Parekh A, et al. Dabigatran versus warfarin in patients with atrial fibrillation. N Engl J Med. 2009;361(12):1139-51. Erratum in: N Engl J Med. 2010;363(19):1877.

12. Mauskopf JA, Sullivan SD, Annemans L, Caro J, Mullins CD, Nuijten M, et al. Principles of good practice for budget impact analysis: report of the ISPOR Task Force on good research practices--budget impact analysis. Value Health. 2007;10(5):33647.

13. Gage BF. Cost of dabigatran for atrial fibrillation. BMJ. 2011;343:d6980.

14. Freeman JM, Zhu RP, Owens DK, Garber AM, Hutton DW, Go AS et al. Cost-effectiveness of dabigatran compared with warfarin for stroke prevention in atrial fibrillation. Ann Intern Med. 2011; 154(1):1-11.

15. Shah S, Gage B. Cost-Effectiveness of dabigatran for stroke prophylaxis in atrial fibrillation. Circulation. 2011;123(22):2562-70.

16. Pink J, Lane S, Pirmohamed M, Hughes D. Dabigatran etexilate versus warfarin in management of non-valvular atrial fibrillation in UK context:quantitative benefit-harm and economic analyses. BMJ 2011;343:d6333.

17. Kamel H, Johnston SC, Easton JD, Kim AS. Cost-effectiveness of dabigatran compared with warfarin for stroke prevention in patients with atrial fibrillation and prior stroke or transient ischemic attack. Stroke. 2012;43(3):881-3.

18. Sorensen SV, Kansal AR, Connolly S, Peng S, Linnehan J, Bradley-Kennedy C et al. Cost-effectiveness of dabigatran etexilate for the prevention of stroke and systemic embolism in atrial fibrillation: a Canadian payer perspective. Thromb Haemost. 2011;105(5):908-19.

19. Kansal AR, Sorensen SV, Gani R, Robinson P, Pan F, Plumb JM, et al. Cost-effectiveness of dabigatran etexilate for the prevention of stroke and systemic embolism in UK patients with atrial fibrillation. Heart. 2012;98(7):573-8.
20. Langkilde LK, Bergholdt Asmussen M, Overgaard M. Cost-effectiveness of dabigatran etexilate for stroke prevention in non-valvular atrial fibrillation. Applying RE-LY to clinical practice in Denmark. J Med Econ. 2012;15(4):695-703.

21. González-Juanatey JR, Álvarez-Sabin J, Lobos JM, Martínez-Rubio A, Reverter JC, Oyagüez I, et al. Cost-effectiveness of dabigatran for stroke prevention in non-valvular atrial fibrillation in Spain. Rev Esp Cardiol. 2012;65(10):901-10.

22. National Institute for Health and Clinicall Excellence. Dabigatrán exilate for the prevention of stroke and systemic embolism in atrial fibrillation; 2012. NICE technology appraisal guidance 249. London: NICE; 2012. [Citado el 5 de Marzo de 2013]. Disponible en: www.nice.org.uk/ta249.

23. Swedish Council on Health Technology Assessment. Dabigatran to Prevent Stroke in Patients With Atrial Fibrillation. SBU Alert Report. 2011. [citado el 5 de Marzo de 2013]. Disponible en: http://www.sbu.se/upload/Publikationer/Content1/1/Dabigatran Prevent Stroke Patients With _Atrial_Fibrillation_201104.pdf

24. Australian Government. Departament of Health and Ageing. Review of Anticoagulation Therapies in Atrial Fibrillation. Commonwealth of Australia; 2012. [citado el 5 de Marzo de 2013].Disponible en: http://www.pbs.gov.au/reviews/atrial-fibrillationfiles/report-anticoagulation.pdf

25. Canadian Agency for Drugs and Technologies in Health. New Oral Anticoagulants for the Prevention of Thromboembolic Events in Patients with Atrial Fibrillation. CADTH; 2012. CADTH Therapeutic Review. [citado el 5 de Marzo de 2013].Disponible en: http://www.cadth.ca/media/pdf/tr0002_NewOral-Anticoagulants_rec_e.pdf

26. Agencia Española de Medicamentos y Productos Sanitarios. Criterios y recomendaciones generales para el uso de nuevos anticoagulantes orales en la prevención del ictus y la embolia sistémica en pacientes con fibrilación auricular no valvular. Informe de posicionamiento terapéutico UT/V2/18122012. Madrid: Ministerio de Sanidad, Servicios Sociales e Igualdad; 2012. [citado el 5 de Marzo de 2 0113 . Disponible en: http://www.aemps.gob.es/medicamentosUsoHumano/informesPublicos/docs/criterios-anticoagulantes-orales_UT_V2_18122012.pdf

27. Mak KH. Coronary and mortality risk of novel oral antithrombotic agents: a meta-analysis of large randomised trials. BMJ Open. 2012;2(5). 
28. Avorn J. The relative cost-effectiveness of anticoagulants: obvious, except for the cost and the effectiveness. Circulation. 2011;123(22):2519-21.

29. Radecki R. Dabigatran-Uncharted waters and potential harms. Ann Intern Med. 2012;157(5):388.

30. Wallentin L, Yusuf S, Ezekowitz MD, Alings M, Flather M, Franzosi MG, et al. Efficacy and safety of dabigatran compared with warfarin at different levels of international normalised ratio control for stroke prevention in atrial fibrillation: an analysis of the RE-LY trial. Lancet. 2010;376(9745):975-83.

31. Roncalés FJ. Oral anticoagulants: warfarin or acenocumarol? Med Clin (Barc). 2008;131(3):98100. 